Supporting Information

\title{
Analysis of the Thermal Behavior of Polypropylene - Camphor Mixtures for Understanding the Pathways to Polymeric Membranes via Thermally Induced Phase Separation
}

Konstantin V. Pochivalov, ${ }^{\mathrm{a}}$ Andrey V. Basko, ${ }^{\mathrm{a}}$ Tatiana N. Lebedeva, ${ }^{\mathrm{a}}$ Anna N. Ilyasova, ${ }^{\mathrm{a}}$ Roman Yu. Golovanov , ${ }^{\text {a }}$ Mikhail Yu. Yurov , ${ }^{\text {a }}$ Georgiy A. Shandryuk, ${ }^{\mathrm{b}}$ Vladimir V. Artemov, Alexander A. Ezhov, ${ }^{\text {b,c,d }}$ Yaroslav V. Kudryavtsev*,b,e

${ }^{a}$ Krestov Institute of Solution Chemistry, Russian Academy of Sciences, Akademicheskaya ul. 1, Ivanovo, 153045 Russia

b Topchiev Institute of Petrochemical Synthesis, Russian Academy of Sciences, Leninskii pr. 29, Moscow, 119991 Russia

c Shubnikov Institute of Crystallography, Federal Scientific Research Centre "Crystallography and Photonics”, Russian Academy of Sciences, Leninskii pr. 59, 119333, Moscow, Russia

d Faculty of Physics, Lomonosov Moscow State University, Leninskie gory 1-2, Moscow 119991 Russia

e Frumkin Institute of Physical Chemistry and Electrochemistry, Russian Academy of Sciences, Leninskii pr. 31, Moscow, 119071 Russia 


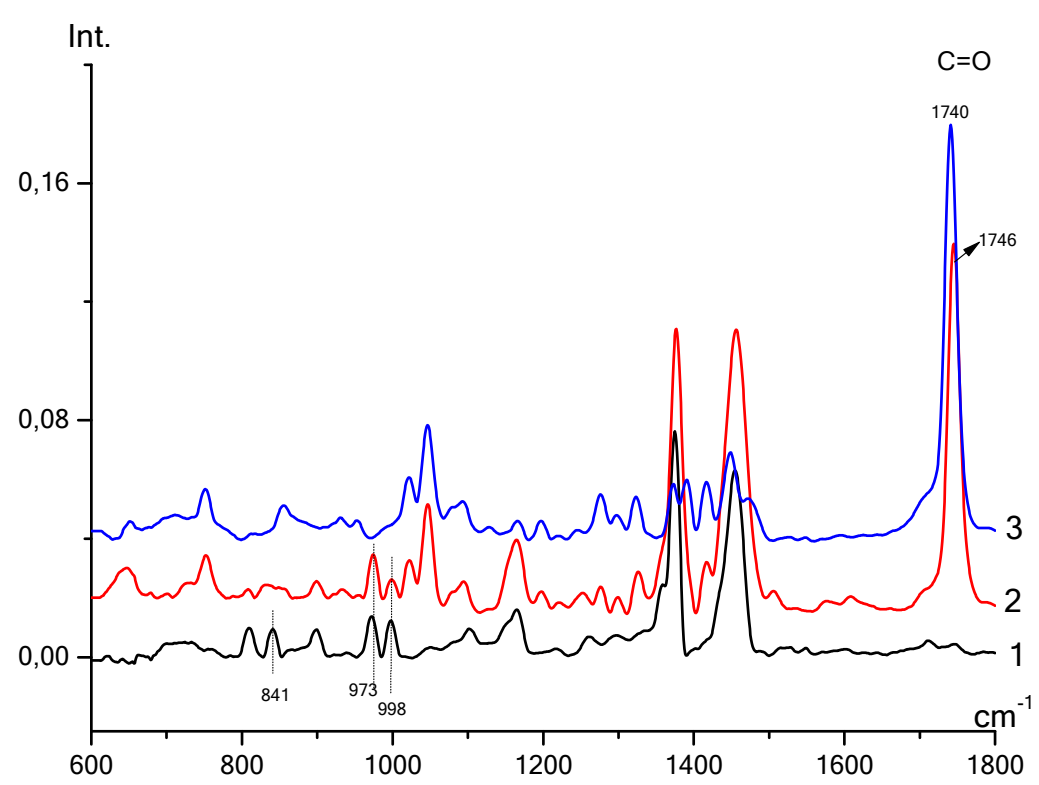

Fig. S1. FTIR reflectance spectra for the films obtained from (1) polypropylene, (2) polypropylene camphor mixture containing ca. $40 \mathrm{wt} \%$ of the polymer and for (3) camphor crystals.

Films of ca. $50 \mu \mathrm{m}$ thickness were prepared by heating polypropylene (PP) or its mixture with camphor up to $200{ }^{\circ} \mathrm{C}$, keeping it at that temperature for $30 \mathrm{~min}$, placing the melt between polyimide substrates, and cooling it down to room temperature. FTIR spectra were recorded in attenuated total reflectance (ATR) mode on a Bruker HYPERION-2000 IR microscope coupled with a Bruker IFS 66v/S spectrometer (Ge crystal, 50 scans, resolution of $2 \mathrm{~cm}^{-1}$, range of $600-4000 \mathrm{~cm}^{-1}$ ). The data were analyzed using the OPUS software package (Bruker). The spectrum of PP - camphor mixture (curve 2 in Fig. S1) is not a superposition of the PP (curve 1) and camphor (curve 3) spectra. In particular, the carbonyl stretching band of camphor is shifted in the mixture spectrum by $5 \mathrm{~cm}^{-1}$ toward short wavelengths. Noticeable changes in the intensity ratios of the individual absorption bands are visible in the PP spectrum as well. For instance, the bands between 800 and $1000 \mathrm{~cm}^{-1}$ are affected, which are sensitive to the polymer microtacticity [Quynn, R. G.; Riley, J. L.; Young, D. A.; Noether, H. D. Density, crystallinity, and heptane insolubility in isotactic polypropylene. J. Appl. Polymer Sci. 1959, 2, 166 - 173. DOI: 10.1002/app.1959.070020506]. Table S1 shows the absolute and relative intensities of the bands reflecting the presence of isotactic $\left(998 \mathrm{~cm}^{-1}\right.$ and $\left.841 \mathrm{~cm}^{-1}\right)$ and atactic $\left(973 \mathrm{~cm}^{-1}\right)$ 
sequences in PP. It is seen that the relative intensity of those bands in pure PP and in its mixture with camphor differ about two times. Thus, it can be concluded that significant conformational changes occur both in PP and in camphor as a result of their mixing.

Table S1. Absolute and Relative Intensities of Several Individual IR Bands

\begin{tabular}{c|c|c|c|c|c} 
Sample & $D_{998}$ & $D_{973}$ & $D_{841}$ & $D_{998} / D_{973}$ & $D_{841} / D_{973}$ \\
Polypropylene & 1.23 & 1.38 & 0.95 & 0.89 & 0.69 \\
$\begin{array}{c}\text { Polypropylene-camphor } \\
\text { mixture }\end{array}$ & 0.74 & 1.77 & 0.49 & 0.42 & 0.28 \\
\hline
\end{tabular}




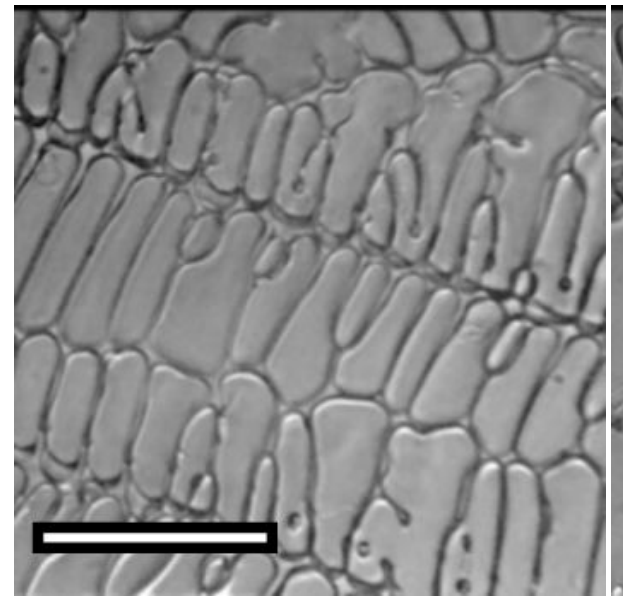

(a)

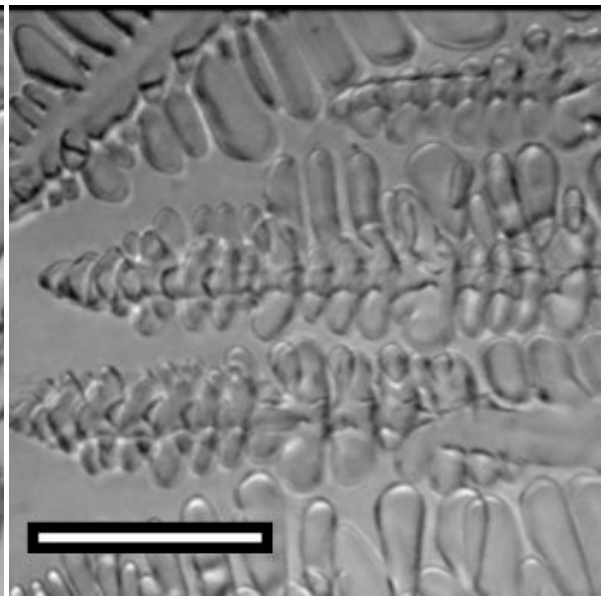

(b)

Fig. S2. Optical micrographs illustrating the shape of camphor crystals formed in the PP - camphor mixture with $15 \mathrm{wt} \%$ of the polymer. Scale bar $=100 \mu \mathrm{m}$.

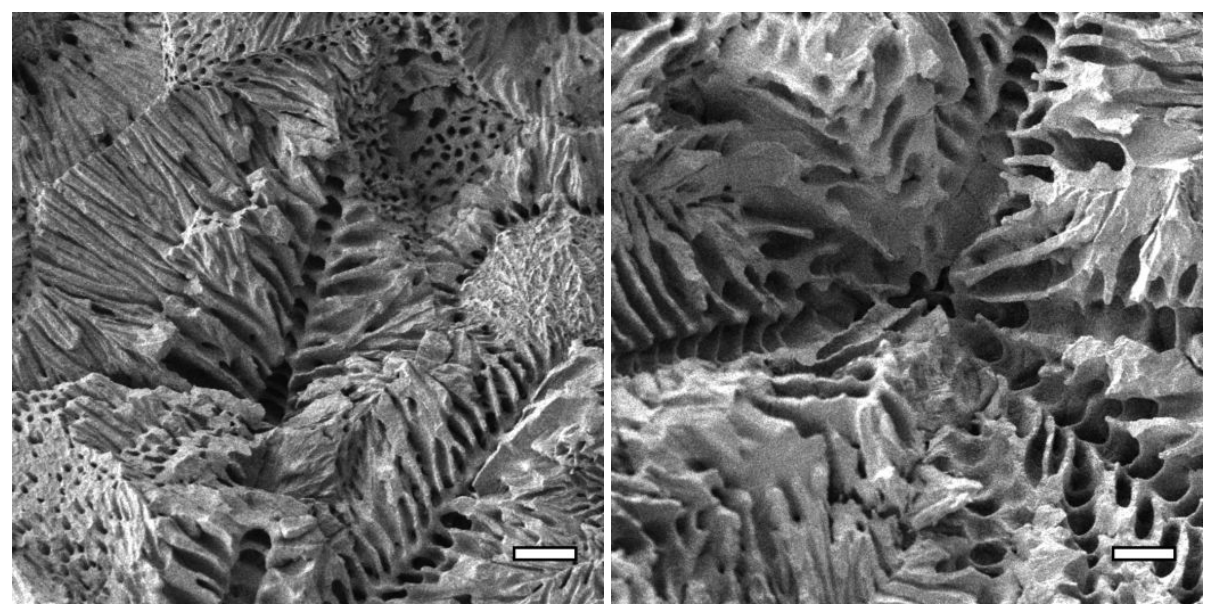

(a)

(b)

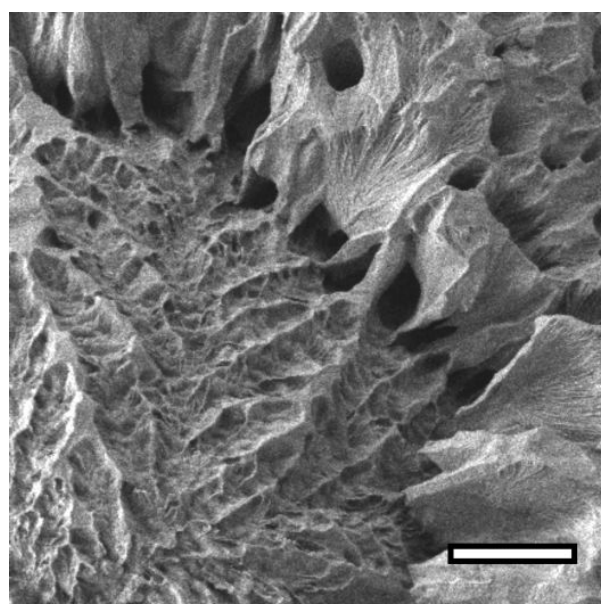

(c)

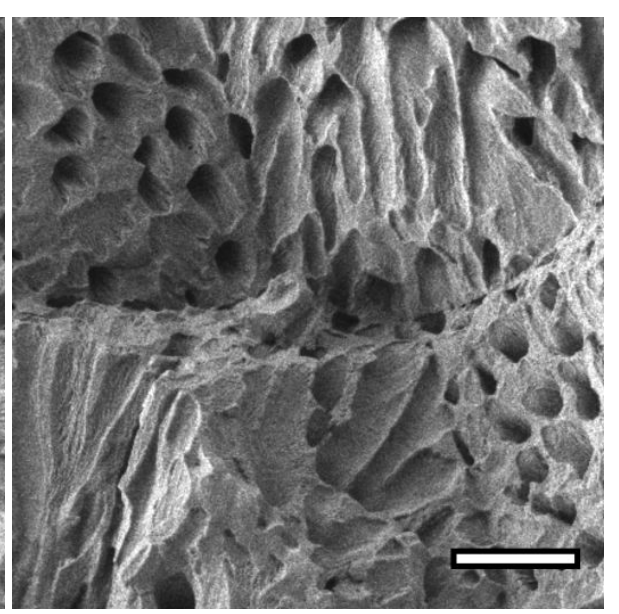

(d) 
Fig. S3. SEM images from different regions of the cleaved membrane obtained via TIPS at a cooling rate of $10{ }^{\circ} \mathrm{C} \mathrm{min}-1$ from the PP - camphor mixture containing $61 \mathrm{wt} \%$ of PP. Scale bars $=(\mathrm{a}, \mathrm{b}) 10$ $\mu \mathrm{m}$ and (c, d) $5 \mu \mathrm{m}$. 


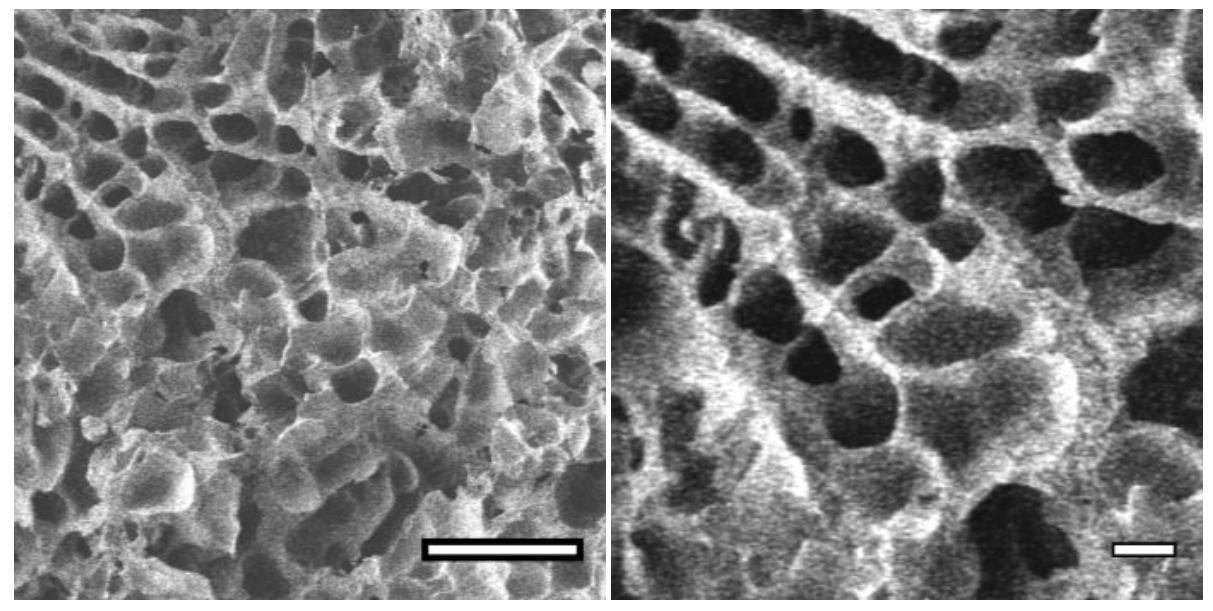

(a)

(b)

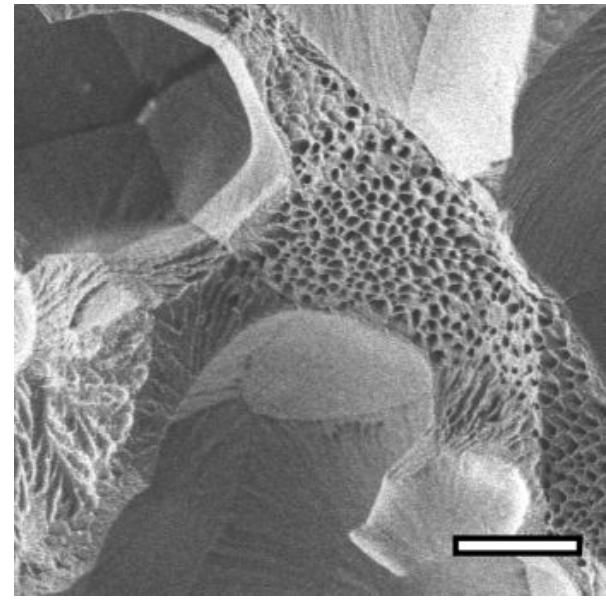

(c)

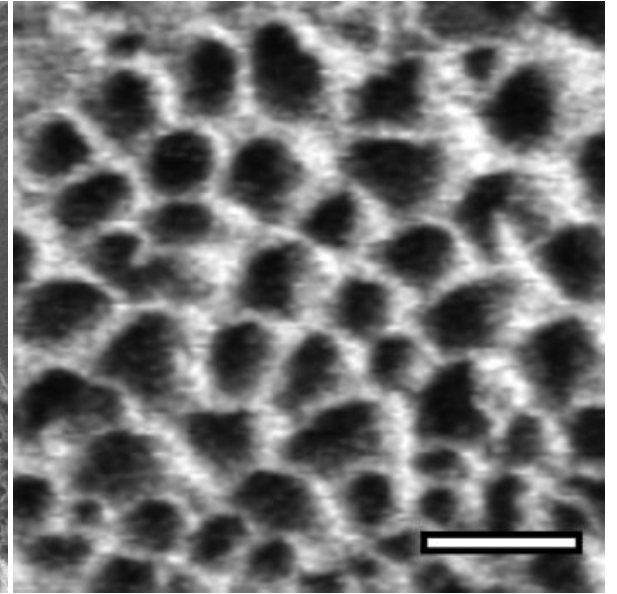

(d)

Fig. S4. SEM images from the cleaved membrane obtained via TIPS at a cooling rate of $200{ }^{\circ} \mathrm{C} \min ^{-1}$ from the PP - camphor mixture containing $26 \mathrm{wt} \%$ of PP. Scale bars $=$ (a) $50 \mu \mathrm{m}$, (b) $10 \mu \mathrm{m}$, (c) $5 \mu \mathrm{m}$, and (d) $1 \mu \mathrm{m}$. 


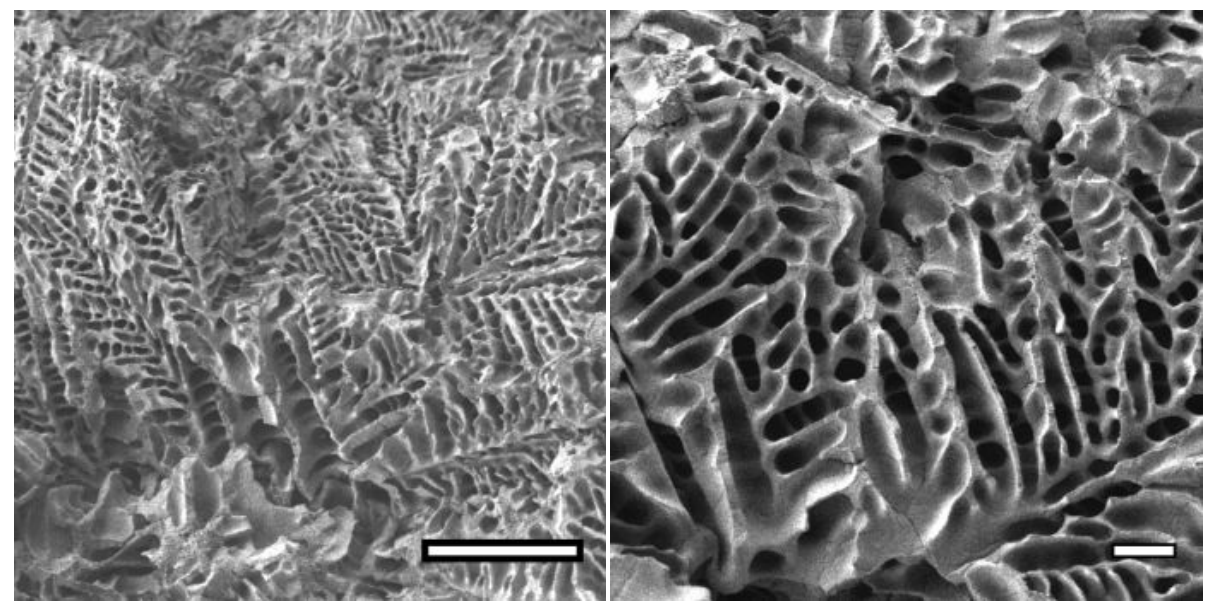

(a)

(b)

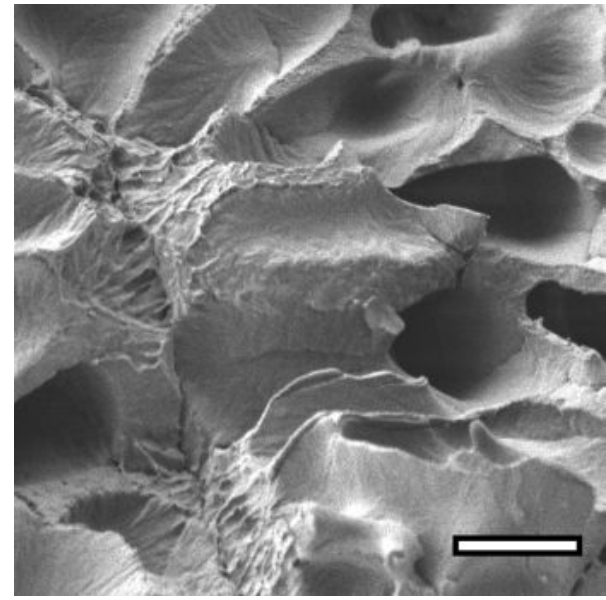

(c)

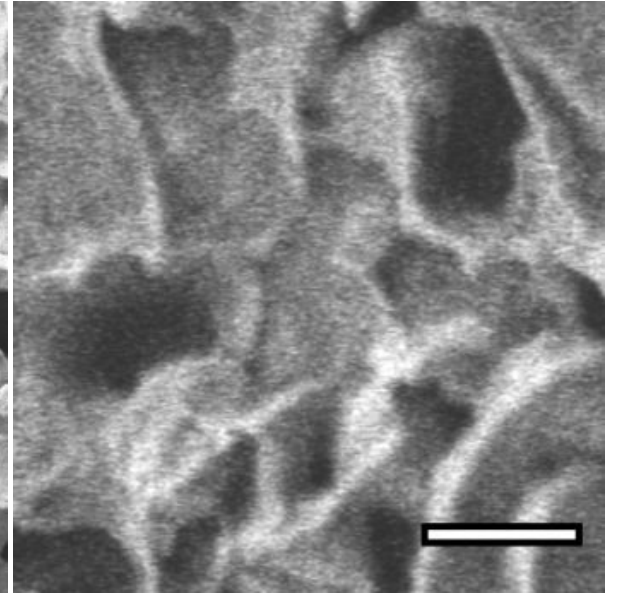

(d)

Fig. S5. SEM images from the cleaved membrane obtained via TIPS at a cooling rate of $200{ }^{\circ} \mathrm{C} \min ^{-1}$ from the PP - camphor mixture containing $41 \mathrm{wt} \%$ of PP. Scale bars $=(\mathrm{a}) 50 \mu \mathrm{m}$, (b) $10 \mu \mathrm{m}$, (c) $5 \mu \mathrm{m}$, and (d) $1 \mu \mathrm{m}$. 


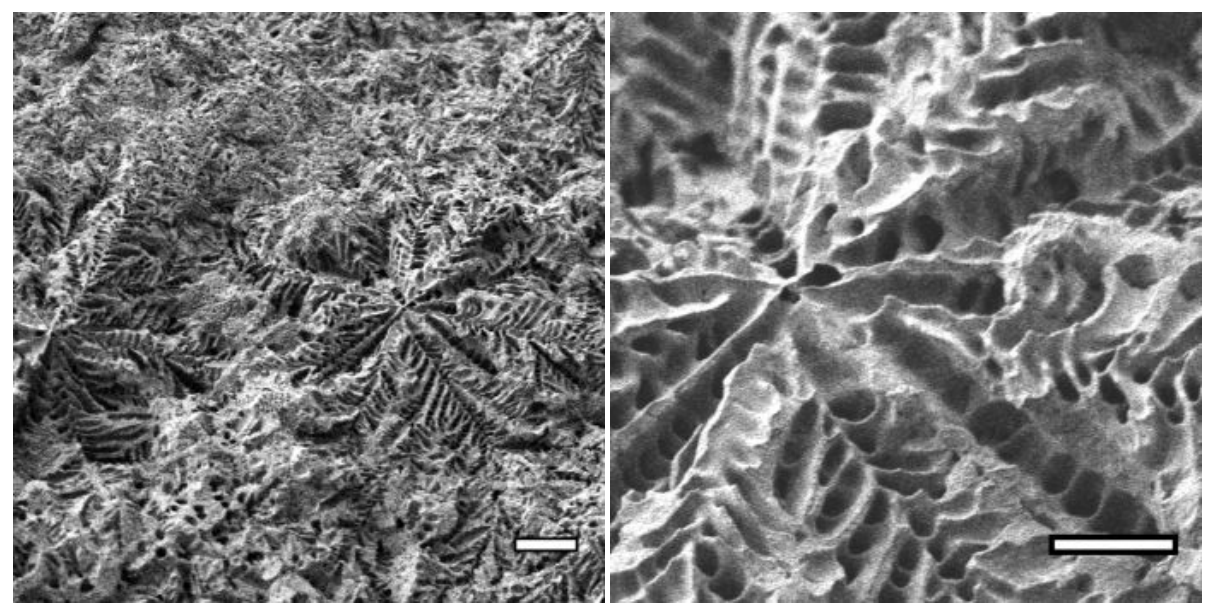

(a)

(b)

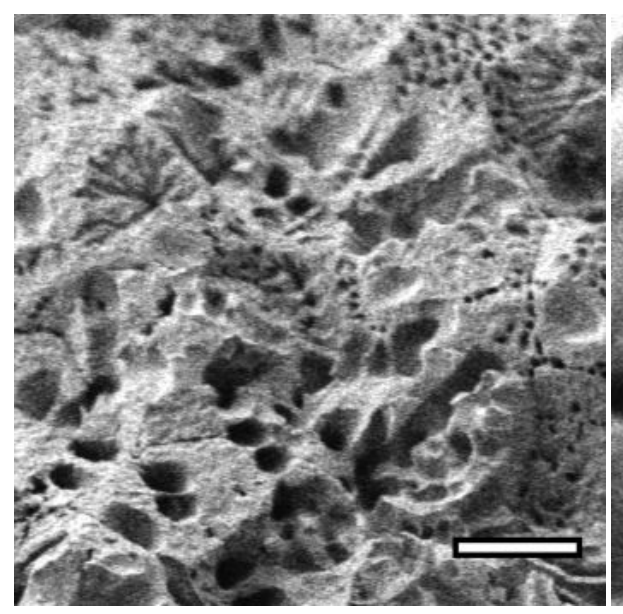

(c)

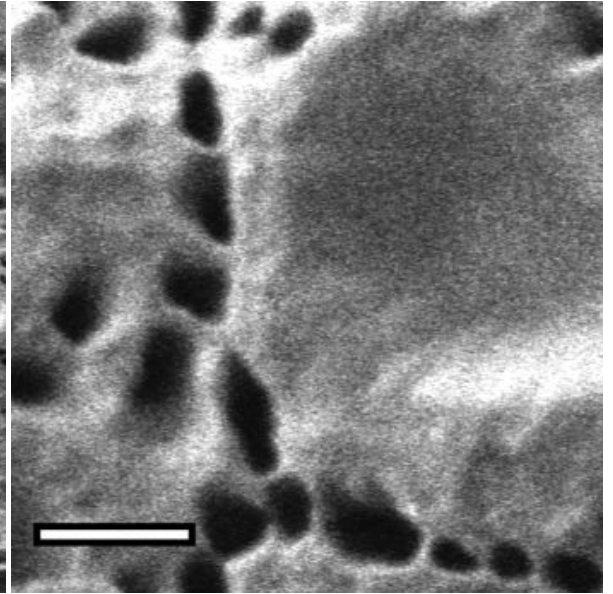

(d)

Fig. S6. SEM images from the cleaved membrane obtained via TIPS at a cooling rate of $200{ }^{\circ} \mathrm{C} \min ^{-1}$ from the PP - camphor mixture containing $57 \mathrm{wt} \%$ of PP. Scale bars $=(\mathrm{a}) 10 \mu \mathrm{m},(\mathrm{b}, \mathrm{c}) 5 \mu \mathrm{m}$, and (d) $1 \mu \mathrm{m}$. 


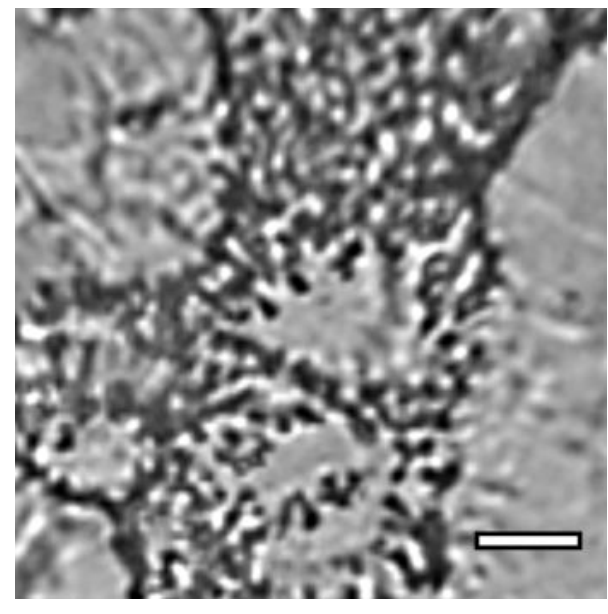

(a)

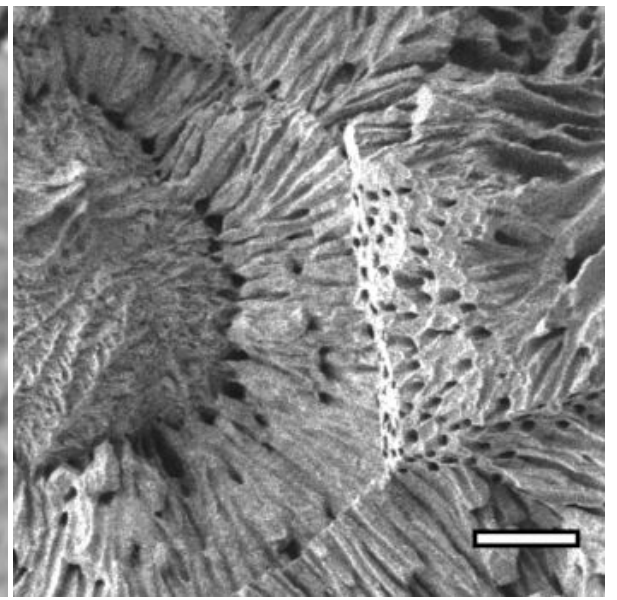

(b)

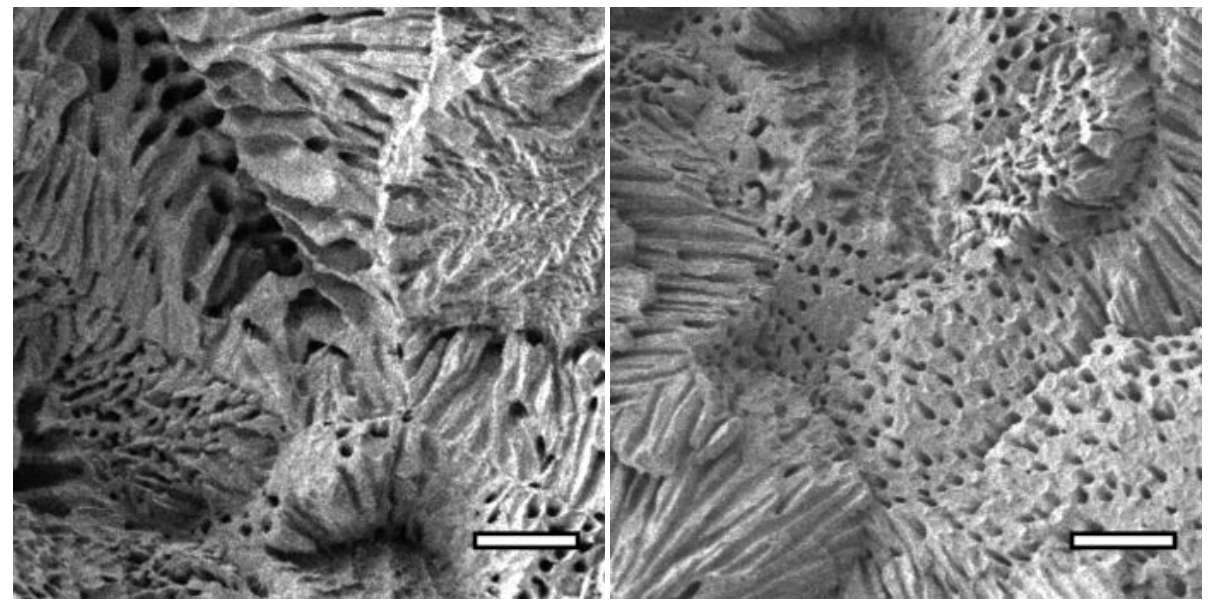

(c)

(d)

Fig. S7. The comparison of (a) optical micrograph of the PP - camphor mixture (60 wt $\%$ of PP) and $(b-d)$ SEM images of the cleaved membrane obtained from it via TIPS at a cooling rate of $10{ }^{\circ} \mathrm{C}$ $\min ^{-1}$. The same magnification is used for all pictures. Scale bar $=10 \mu \mathrm{m}$. 